\section{New topical therapies}

\section{Introduction}

Topical medications remain a cornerstone in dermatologic therapeutic strategies for many reasons. It is easily applied in most cases (except for back lesions), with relative rapid response and potentially less systemic side effects compared to oral and injectable medicines. In the last two years many topical therapies were investigated, some were approved while others are still in trials. In this short article some of the most recent topical agents will be discussed briefly under disease categories: hyperhidrosis, acne vulgaris, alopecia areata and epidermolysis bullosa.

\section{Hyperhidrosis}

Topical anticholinergic Glycopyrronium tosylate was approved by FDA in June 2018. It is developed for once daily use to treat primary auxiliary hyperhidrosis. Qbrexa (pronounced kew brex'zah) is sold commercially as individually packed cloth or swipes. It is self administered home medication that may be applied by any patient 9 years old and above. Side effects included local erythema (17\%), burning or stinging (14\%) and dry mouth in one fifth of the patients. ${ }^{1,2}$

\section{Acne vulgaris}

Acne is still of a great focus in clinical trials. We will discuss three new topical agents. The first is named Cortexolone 17 alpha propionate $1 \%$ cream and is now in a phase 3 on 1400patients. It is a potent topical selective testosterone inhibitor; but has no effect on 5 alpha reductase enzyme. It has no systemic side effects on gonads as well as on the adrenal axis. ${ }^{3-5}$ The second anti-acne topical agent is considered if approved a historic drug. It is the first topical sebum production inhibitor Olumacostat glasaretil. It is tried in a gel (nongreasy) form. Results of phase 3 trial was released in May 2018, but was behind initial great expectations. ${ }^{6,7}$ The third anti-acne is named SB204, it is a nitric oxide-releasing topical gel. It possesses immunemodulatory and antibacterial effects. It already met the clinical endpoints in two phase 3 studies and has been prepared for submission to FDA. ${ }^{8,9}$

\section{Alopecia areata}

Topical Janus kinase inhibitors mainly tofacitinib ${ }^{\circledR}$ and ruxolitinib ${ }^{\circledR}$ were investigated in many studies that include different stages, severity indices and age groups of patients with alopecia areata. Results were conflicting; however, still the group is probed because alopecia areata has only limited therapeutic options. Topical JAK inhibitors while showing modest effect in alopecia still show promising result in other skin conditions as psoriasis and stopic eczema. ${ }^{10}$

\section{Epidermolysis bullosa simplex (EBS)}

It is a rare genetic disorder with (unfortunately) almost no effective treatment. New molecular technology leads to discovery of the mechanism of the mechanical-induced cytolysis. It revealed that mechanical stress on abnormal keratin K5/14 leads to activation of IL-1 $\beta$, which cause cytolysis and leads to enhancement for formation of more abnormal K5/14 as a positive feedback. ${ }^{11}$ Diacerein is a prodrug that is transformed into IL-1 $\beta$ converting enzyme inhibitor that decreases the level of IL-1 $\beta$ and abnormal K5/14. Blister formation is reduced significantly, as compared to placebo within
Volume 2 Issue 5 - 2018

\author{
Hassan Riad \\ University of Ain Shams, Egypt
}

Correspondence: Hassan Riad, Designation Prof. Dr., University of Ain Shams, Abbasya Cairo, Egypt Tel +20I0 9877 7848, Email hssnrd@yahoo.com

Received: August 18, 2018| Published:September 26, 2018

two weeks and remained significantly below the baseline after cream discontinuation. ${ }^{12}$

\section{Conclusion}

In conclusion, we are lucky to witness this era of evolution of new topical therapies for diseases that were considered intractable and hard to manage in the past, seem on a promising path.

\section{Acknowledgements}

None.

\section{Conflict of interest}

Author declares that there is conflict of inertest.

\section{References}

1. Kluger N, Saarinen K. Aspergillus fumigatus infection on a home-made tattoo. Br J Dermatol. 2014;170(6):1373-1375.

2. Glaser DA, Hebert AA, Nast A, et al. Topical Glycopyrronium Tosylate for the Treatment of Primary Axillary Hyperhidrosis: Results from the ATMOS-1 and ATMOS-2 Phase 3 Randomized Controlled Trials. J Am Acad Dermatol. 2018; pii: S0190-9622(18)32224-32222.

3. Trifu V, Tiplica GS, Naumescu E, et al. Cortexolone $17 \alpha$-propionate $1 \%$ cream, a new potent antiandrogen for topical treatment of acne vulgaris. A pilot randomized, double-blind comparative study vs. placebo and tretinoin 0.05\% cream. Br J Dermatol. 2011;165(1):177-183.

4. Zouboulis CC, Dessinioti C, Tsatsou F, et al. Anti-acne drugs in phase 1 and 2 clinical trials. Expert Opin Investig Drugs. 2017;26(7):813-823.

5. https://clinicaltrials.gov/ct2/show/NCT02682264

6. Melnik BC. Olumacostat Glasaretil, a Promising Topical SebumSuppressing Agent that Affects All Major Pathogenic Factors of Acne Vulgaris. J Invest Dermatol. 2017;137(7):1405-1408.

7. http://investor.dermira.com/news-releases/news-release-details/dermirastwo-phase-3-trials-evaluating-olumacostat-glasaretil

8. https://globenewswire.com/news-release/2018/02/13/1339810/0/en/ Novan-to-Present-Integrated-Data-from-Phase-3-Trials-with-SB204-forTreatment-of-Acne.html

9. Eichenfield LF, Gold LS, Nahm WK, et al. Results of a Phase 2, Randomized, Vehicle-Controlled Study Evaluating the Efficacy, Tolerability, and Safety of Daily or Twice Daily SB204 for the Treatment of Acne Vulgaris. J Drugs Dermatol. 2016;15(12):1496-15027. 
10. Putterman E, Castelo-Soccio L. Topical 2\% tofacitinib for children with alopecia areata, alopecia totalis, and alopecia universalis. $J$ Am Acad Dermatol. 2018;78(6):1207-1209.

11. Wally V, Lettner T, Peking PJ, et al. The pathogenetic role of IL$1 \beta$ in severe epidermolysis bullosa simplex. J Invest Dermatol. 2013;133(7):1901-1903.
12. Wally V, Hovnanian A, Ly J, et al. Diacerein orphan drug development for epidermolysis bullosa simplex: A phase 2/3 randomized, placebo-controlled, double-blind clinical trial. J Am Acad Dermatol. 2018;78(5):892-901. 